УДК 81

DOI: 10.33184/NYVB-2021-04-06.11

Steppan, Andreas

(Oberstudienrat, Fachschaftsberater (Deutschland), Gymnasium Nr. 86 Ufa)

\title{
ZUM STAND DER WISSENSCHAFTLICHEN ERFORSCHUNG DER DEUTSCHSPRACHIGEN LITERATUR POST- SOWJETISCHER MIGRANTEN ODER «FREMD IST DER MIGRANT IN JEDEM LAND»
}

In diesem Artikel geht es um ein Segment der aktuellen deutschsprachigen Literatur, das im vergangenen Jahrzehnt große Popularität erzielte. Dabei handelt es sich um Autorinnen und Autoren, die als biografische Besonderheit einen (post)sowjetischen (Migrations)Hintergrund haben (ohne Anspruch auf Vollständigkeit: Alina Bronsky, Olga Grjasnowa, Lena Gorelik, Eugen Ruge, Sasha Marianna Salzmann, Olga Martynova, Irina Liebmann, Nino Haratischwili, Lana Lux, Wlada Kolosowa, Katerina Poladjan, Marina Frenk, Eleonora Hummel, Wladimir Kaminer, Dmitrij Kapitelman, Natascha Wodin, Vladimir Vertlib, Dmitrij Wall und andere).

\section{Fragestellung}

Für wen schreibt der Autor? ist eine Frage, die sich die Literaturwissenschaft seit jeher stellt, im Gegensatz zum Autor selbst und meist auch zu seinem Publikum. Es geht bei ihr also nicht um eine intentionale Kategorie, sondern um eine distanzierte Analyse, die den Selbstaussagen der Beteiligten weniger traut als den Signalen des Textes (die von einem hermeneutischen oder semiotischen Standpunkt aus interpretiert werden) und der um ihn als Botschaft herum sich entwickelnden Kommunikation (die literatursoziologisch oder ebenfalls semiotisch interpretiert wird). Indem beide Ansätze verbunden werden, ergibt sich eine Relation zwischen den Textsignalen und der (realen) Leserschaft. Im besten Falle kann die Forschung dadurch beantworten, welche Textsignale welche Leserschaft ansprechen und den kommerziellen Erfolg eines Werkes begründen. 


\section{Begründung}

Während sich die Autorinnen und Autoren dieser (bislang nicht befriedigend definierten oder benannten) Gruppe teils vehement dagegen aussprechen, aufgrund dieses zufälligen biografischen Details als eine Gruppe wahrgenommen zu werden, ist die Existenz einer Leserschaft, die gerade hierin einen entscheidenden Faktor sieht, nicht zu bezweifeln. Wenn man sich die begleitende Verlagswerbung ansieht, muss man zu dem Schluss kommen, dass die Zugehörigkeit des Autors zur Gruppe der „Ost-West-Migranten“ den Verkauf des Produktes positiv beeinflusst.

\section{Hilfsmittel quantitativ}

Besonders interessant sind diese bikulturellen Autoren zunächst für die deutschen Slawisten und die russischen Germanisten, weniger für die deutschen Germanisten, da an der deutschen Universität nach wie vor das System der Nationalphilologien vorherrscht. Hier ist die Herkunft oder der Hintergrund des Autors tatsächlich eher eine individuelle biografische Fußnote. Eine literatursoziologische Fragestellung wird zudem erschwert durch die intransparente Auflagenhöhe. Es gibt sowohl quantitative (Verkaufszahlen insgesamt, Leserbefragungen) als auch qualitative (Feuilleton, Literatursendungen, Literaturpreise, Bestenlisten) Daten, aber keine Kopplung dieser Daten, die z. B. Auskunft über die tatsächliche Leserschaft eines bestimmten Buches geben. So gehen noch nicht einmal aus den Bestsellerlisten (z. B. des SPIEGEL) absolute Zahlen hervor, geschweige denn eine Auffächerung nach Sinusmilieus oder anderen gesellschaftlichen Gruppen.

Dennoch sind gerade die Spiegel-Bestsellerlisten aufschlussreich, indem sie nach zwei Seiten wirken: einmal bilden sie die gesamtgesellschaftliche Tendenz $\mathrm{zu}$ bestimmten Themen $\mathrm{ab}$, andererseits verbessert die Aufnahme eines Titels in diese Liste seine weiteren Verkaufschancen. Leider liegen hierfür aber nur die Top 15 oder Top 20 vor, unter denen sich dann wirklich nur die ganz Großen wiederfinden. Die Offenlegung der Top 100 mit absoluten Verkaufszahlen wäre hier äußerst nützlich, um annähernd realistisch die Breitenwirkung eines Titels abzuschätzen. 


\section{Forschungsstand}

Trotz dieser ungünstigen und dem Selbstverständnis der deutschen Germanistik eher widersprechenden Voraussetzungen hat sich die Forschung in der letzten Zeit verstärkt dieser Autorengruppe angenommen, mit dem Ergebnis einer ersten umfassenden Dissertation von Nora Isterheld an der Universität Bamberg, „In der Zugluft Europas“" (so auch der Titel eines Gedichtbandes von Olga Martynova) im Jahre 2017. In einem jüngst an der Universität Freiburg von Matthias Aumüller und Weertje Willms herausgegebenen Sammelband („Migration und Gegenwartsliteratur“, Paderborn 2020) wird die Perspektive auf andere Länder Osteuropas erweitert. Ich möchte im Folgenden den Stand meiner eigenen Forschungen skizzieren.

Die post-sowjetische deutschsprachige Literatur erzählt von der überwundenen Vergangenheit

Der Jahrtausendwechsel 1999/2000 brachte ein neues Geschichtsverständnis mit sich, das den Begriff „Narrativ“ von der Literaturwissenschaft entlehnte, gleichzeitig aber beklagte, dass die Literatur sich vom klassischen „Erzählen“ wegbewegt habe. Offenbar war das "Narrativ“ der deutschen Teilung noch nicht hinreichend in eine historische Teleologie transzendiert worden. Im Gegensatz zum Film (der schnell das groteske Potenzial für sich entdeckte) tat sich die Literatur noch schwer mit der DDR-Aufarbeitung. Das Gefühl, an einer Zeitenwende zu stehen, ließ sich eher mit Sachbüchern unterfüttern als mit Belletristik. Mit dem furchtbaren 11. September 2001 war überdies der „Kampf der Zivilisationen“ in den Vordergrund gerückt worden und dessen journalistische Aufarbeitung mit Slogans wie ,spannender als ein Krimi“ oder „die jede Phantasie übertreffende Realität“ der literarischen Verarbeitung davongelaufen.

Die post-sowjetische deutschsprachige Literatur strukturiert die diffuse Gegenwart

Für den Blick auf Russland sprachen zu dieser Zeit mehrere Gründe: die wieder ernster zu nehmende Politik des neuen Präsidenten, die gemeinsame Front gegen den Islamismus, die Westöffnung der Ukraine, der Einzug der postsowjetischen Popkultur in die 
westeuropäische, die spürbare Zahl an Spätaussiedlern, die sich über ganz Deutschland verteilt hatten. Und Wladimir Kaminer. Auch wenn die „ernsthafte“ Literaturwissenschaft ihn heute noch mit spitzen Fingern anfasst, wurde er der inoffizielle Nachfolger von Ivan Rebroff, seit 2000 die „Russendisko“ erschienen war. Hinzu kam das nun bereits als Narrativ verstandene Erbe der deutschen Vertriebenen. Paradoxerweise führte das Aussterben der Erlebensgeneration nämlich $\mathrm{zu}$ einer weniger ideologisch aufgeladenen, damit aber breiteren und zustimmungsfähigeren Aufarbeitung der deutschen Geschichte im Osten. Zuletzt und nicht zumindest führte das Bewusstsein vom Jahrtausendwechsel zu der Sehnsucht nach dem Rundumschlag, nach dem „Panorama des 20. Jahrhunderts“, das in der Folge hundertfach als Verkaufsargument herhalten musste.

Die post-sowjetische deutschsprachige Literatur ist kommerziell relativ erfolgreich

Aus dem engeren Kreis der hier behandelten Autoren schaffte es in die Jahresbestsellerlisten nur der im Gebiet Sverdlovsk geborene Eugen Ruge (mit „In Zeiten des abnehmenden Lichts“, Platz 7/2011). Bereits 2007 erreichte die in Kiel geborene, jetzt aber in England lebende (und auf Englisch schreibende, weshalb ich sie nicht direkt zu dieser Gruppe zähle) Tochter ukrainischer Eltern, Marina Lewycka (mit der aus dem Englischen übersetzten „Kurzen Geschichte des Traktors auf Ukrainisch") einen bahnbrechenden siebten Platz in der Jahresbestsellerliste.

Eine Recherche über das Bestsellerarchiv von Buchreport, dem ausführenden Partner des Spiegels, ergibt noch ein halbes Dutzend weiterer Autoren, die sich großenteils unterhalb der Top 20 kurzzeitig in den verschiedenen neu hinzugekommenen Listen aufhielten (vor allem Taschenbuchausgaben, Hörbücher und Verfilmungen). Eine Ausnahme stellt nur der bereits erwähnte Wladimir Kaminer dar, der durch seine humoristischen Gegenwartsporträts offenbar bereits Kultstatus erreicht hat und auch den weniger kulturbeflissenen Fernsehzuschauern bekannt ist, da er gern gesehener Gast in teils launigen, zunehmend aber durchaus ernsthaften Talkshows ist. Einmal 
in den letzten 20 Jahren, nämlich 2010, wurde zu einem Siegertitel der Jahresbestsellerliste (Thilo Sarrazins ,Deutschland schafft sich ab“) die Verkaufszahl 1,2 Millionen genannt. Es ist anzunehmen, dass das eine besonders eindrucksvolle Zahl ist, sodass man vielleicht als Größenordnung für einen ersten Platz einen Jahresabsatz von 1 Million Exemplaren ansetzen kann. Dabei handelt es sich allerdings um die Sachbuch-Liste, die vermutlich ohnehin ein größeres Publikum abbildet. Geteilt durch 52 käme man auf durchschnittlich rund 20.000 verkaufte Exemplare wöchentlich, was vermutlich für die allermeisten der von uns hier betrachteten Texte deutlich mehr als die langjährige Gesamtauflage darstellt.

Die Literaturkritik steht der post-sowjetischen deutschsprachigen Literatur freundlich gegenüber

Kommen wir zu den qualitativen Daten. Bezeichnend ist eine Rezension auf Spiegel-Online (4.10.2014) des Romans „Das achte Leben (für Brilka)“ von Nino Haratischwili, den der Rezensent als ,in mancherlei Hinsicht den eigentlichen Roman des Jahres“ feiert, wohlgemerkt ohne eine einzige Nennung dieses Romans in den Spiegel-Bestsellerlisten des ganzen Jahres. Die beste Platzierung erreicht dieser Liebling der Buchhändler erst in Heft 5/2015 laut Buchreport mit Platz 38.

\section{Verkaufsargumente und literarische Qualität.}

Dieses Werk ist ,in mancherlei Hinsicht“ ebenso ein Höhe- wie Wendepunkt der Literatur der post-sowjetischen Migranten. Die Rezensionen sind überwiegend freundlich, aber nicht euphorisch. Am schnell nachgeschobenen Tschetschenien-Wälzer „Die Katze und der General" wird deutlich, dass der Trend zum Kolossalgemälde die Schwächen des Genres offenlegt. „Mehrjährige Recherche“ gesellt sich als Quasi-Werturteil zum „Panorama des 20. Jahrhunderts“, wird aber, wo ein über die Trivialliteratur hinausgehender Stilwille fehlt, schnell zum Manko. An Olga Grjasnowas aktuellem Kaukasusbuch „Der verlorene Sohn“ werden (im Deutschlandfunk Kultur vom 8.9.2020) uninspiriert abgearbeitete Recherchen und klischeehafte Figurenzeichnung bemängelt, womit die Grenze zum Kitsch überschritten wäre. 


\section{Publikumsgeschmack und Autorenintention}

Der relative Erfolg der postsowjetischen Migrantenliteratur hängt wahrscheinlich mit dieser Nähe zum Kitsch zusammen, denn die große Mehrheit der Leser entscheidet sich im Zweifel eben doch für die schöne Geschichte und nicht für die quälende Realitätsrecherche. Dennoch gibt es auch diese, z. B. bei Eleonora Hummel oder Sasha Marianna Salzmann. Wir kommen hier zu einem letzten Problemkomplex: der Zugehörigkeit der post-sowjetischen deutschsprachigen Autoren zu verschiedenen Migrantengruppen.

Status der Zuwanderer und Erwartung der Leser

Die zwei größten Migrantengruppen aus der ehemaligen Sowjetunion sind die Spätaussiedler und die Kontingentflüchtlinge. Aussiedler oder Spätaussiedler sind die Russlanddeutschen, Kontingentflüchtlinge die sowjetischen Juden. Während das deutsche Publikum mit den Russlanddeutschen auch heute noch eher eine gewisse Provinzialität verbindet (bäuerlich und heimatverbunden), erwartet es von den jüdischen Autoren im Gegenteil eine gewisse Weltläufigkeit, den jiddischen Witz oder eine für Nichtjuden unverständliche KabbalaMystik. Es gibt deutlich mehr jüdische als russlanddeutsche Autoren unter den erfolgreichen Titeln. Die dritte und statistisch kleinste Gruppe wird von den Russen oder anderen Nationalitäten der ehemaligen Sowjetunion gebildet, die zu keiner der ersten beiden Gruppen gehören und die es aus anderen Gründen in die Bundesrepublik verschlagen hat. Hinzu treten Kinder sowjetischer Eltern, die bereits in Deutschland geboren wurden und deren Eltern zu einer der drei Gruppen gehören. Eine Sondergruppe unter diesen nehmen wiederum die Kinder von Zwangsarbeitern während des Dritten Reichs ein, die in Deutschland geboren, aber nach dem Krieg als displaced persons eingestuft wurden.

\section{Übersetzte Literatur}

Alle diese Autoren schreiben auf Deutsch (eine Voraussetzung für ihre Zugehörigkeit zur Gruppe). Neben ihnen gibt es ein Segment von unklarer Größe, das als Exilliteratur im engeren Sinne beschrieben werden kann. Es sind vorwiegend russlanddeutsche Dichter, die auf Russisch für ein russlanddeutsches Publikum schreiben. Einige 
Emigranten der anderen beiden Gruppen schreiben zwar auf Russisch, nehmen aber selbst aktiv an der Übersetzung ins Deutsche teil. Ebenfalls und vielleicht noch in höherem Maße als die Genannten finden russische Autoren oder solche aus den ehemaligen Sowjetrepubliken ihr deutsches Publikum durch Übersetzungen, die mit ähnlicher Begleitmusik beworben werden wie deutsch geschriebene Literatur.

\section{Russische Gegenwartsliteratur auf dem deutschen Buchmarkt}

So passt Gusel Jachina ihre „Wolgakinder“ so stark an ihr deutsches Publikum an, dass sie speziell für die deutsche Übersetzung das letzte Kapitel verändert und erweitert hat. Viktor Martinowitschs auf Russisch verfasste „Revolution“ wird als Kommentar zur belarussischen Blumenrevolution beworben und vom Übersetzer mit einem kleinen Anmerkungen-Anhang versehen. Ludmila Ulitzkajas Roman der Stunde, „Eine Seuche in der Stadt", stammt, zu schön, um wahr zu sein, noch aus der Zeit der Sowjetunion und kann daher obendrein noch mit prophetischen Gaben punkten, so wie Martinowitsch mit dem Publikationsverbot in seiner Heimat.

\section{Interkulturelle Übersetzung}

Diese verkaufsfördernden außerliterarischen Faktoren verstellen für eine Weile den genuin literarischen Wert dieser Texte, und zwar sowohl in der negativen Hinsicht der Verdunkelung (der Qualität, wenn z. B. der Erfolg schon wieder skeptisch macht) als auch in der positiven der Überstrahlung (von Schwächen durch die einhellige Begeisterung der Kritiker). Es scheint jedenfalls so $\mathrm{zu}$ sein, dass im vergangenen Jahrzehnt das außerliterarische Interesse an Migrationsgeschichten vor allem in Ost-West-Richtung zusammentraf mit einer beachtlichen Zahl talentierter Schriftstellerinnen und Schriftsteller, die sehr schnell eine bedeutende Rolle im Büchermarkt eingenommen haben. Es bleibt abzuwarten, ob das Publikum ihnen auch folgt, wenn sie sich anderen Themen zuwenden.

Dieser Text erscheint wegen des begrenzten Platzes ohne Anmerkungen. Der Artikel mit allen Anmerkungen kann bei mir unter 
fachschaft.ufa@yandex.ru angefordert werden. Auch über Reaktionen und Fragen freue ich mich.

(C) Steppan, A., 2021 г.

НЕМЕЦКОЯЗЫЧНОЕ ОБРАЗОВАНИЕ И ДИАЛОГ КУЛЬТУР

УДК $81(07)$

DOI: 10.33184/NYVB-2021-04-06.12

Алина Т.Н.

(учитель нем.яз. МБОУ «Гимназия № 86», г. Уфа)

\section{KREATIVES SCHREIBEN ALS DIE ART DES KREATIVEN DENKENS}

B статье рассматриваются подходы $\kappa$ формированию креативного мышиения школьников как части мониторинга функциональной грамотности. Обосновывается возможность использования при этом технологии креативного письма. Дана краткая характеристика приемов технологии креативного письма.

Ключевые слова: Eine qualitativ hochwertige Bildung, vergleichende internationale Studien, PISA, kreatives Denken, die funktionale Grundbildung, Kreatives Schreiben, Schreibfähigkeiten, Kompetenzen, Impulse, Schreibanlässe.

Eine qualitativ hochwertige Bildung ist die Aufgabe, mit der die russische Schulbildung konfrontiert war und ist. Der Text des Dekrets des russischen Präsidenten Wladimir Putin von 2018 "Über nationale Ziele und strategische Ziele der Entwicklung der Russischen Föderation für den Zeitraum bis 2024" besagt, dass die Regierung der Russischen Föderation bei der Entwicklung des nationalen Projekts im Bildungsbereich davon ausgehen muss, dass im Jahr 2024 die Erreichung die folgenden Ziele und Vorgaben sichergestellt werden muss: die Gewährleistung der globalen Wettbewerbsfähigkeit der 